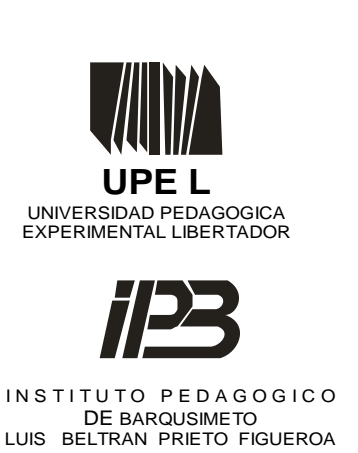

DE BARQUSIMETO
LUIS BELTRAN PRIETO FIGUEROA

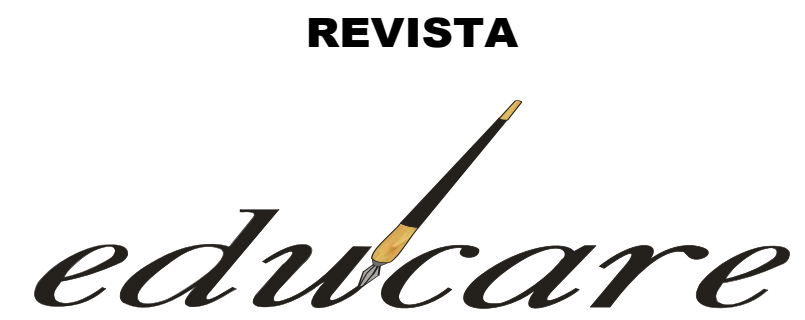

Órgano Divulgativo de la Subdirección de Investigación y Postgrado del Instituto Pedagógico de Barquisimeto "Luis Beltrán Prieto Figueroa"

BARQUISIMETO - EDO. LARA - VENEZUELA

NUEVA ETAPA

FORMATO ELECTRÒNICO

DEPOSITO LEGAL: ppi201002LA3674

ISSN: $2244-7296$

Volumen $18 \mathbf{N}^{\circ} 2$

Mayo - Agosto 2014

\title{
LA UNIVERSIDAD EN LA PERSPECTIVA DE LA INTERDISCIPLINARIEDAD Y TRANSDISCIPLINARIEDAD
}

\section{UNIVERSITY IN THE INTERDISCIPLINARY AND TRANSDISCIPLINARY}

PERSPECTIVE

\section{Rosmel Orfila*}

* Universidad de Carabobo 


\section{LA UNIVERSIDAD EN LA PERSPECTIVA DE LA INTERDISCIPLINARIEDAD Y TRANSDISCIPLINARIEDAD}
UNIVERSITY IN THE INTERDISCIPLINARY AND TRANSDISCIPLINARY PERSPECTIVE

\begin{tabular}{|c|c|}
\hline ENSAYO & Rosmel Orfila." \\
\hline Recibido: 21/01/2014 & Aceptado: 10/05/2014 \\
\hline RESUMEN & ABSTRACT \\
\hline $\begin{array}{l}\text { El presente ensayo trata de poner de } \\
\text { manifiesto un conjunto de reflexiones en } \\
\text { torno al papel de la interdisciplinariedad y } \\
\text { transdisciplinariedad en los procesos de } \\
\text { generación de conocimientos que se } \\
\text { desarrollan dentro de las universidades. } \\
\text { Para lograr una exposición clara, se inicia } \\
\text { con un breve relato en torno a la manera } \\
\text { primitiva de como los seres humanos se } \\
\text { interrelacionaron con el entorno, generando } \\
\text { saberes ancestrales que fueron parte de la } \\
\text { génesis de ulteriores conocimientos. } \\
\text { Seguidamente, se desarrollan argumentos } \\
\text { tendentes a poner en evidencia los } \\
\text { elementos teóricos y discursivos de las } \\
\text { perspectivas interdisciplinarias } \\
\text { transdisciplinarias en el interés de } \\
\text { comprender la naturaleza y dinámica de las } \\
\text { mismas, lo que permitió configurar un } \\
\text { conjunto de aportes cuya pretensión está en } \\
\text { servir para el debate y discusión de ideas } \\
\text { puntuales cuyo norte se circunscribe el abrir } \\
\text { la posibilidad de alcanzar un conocimiento } \\
\text { total y no fragmentado. }\end{array}$ & $\begin{array}{l}\text { This essay tries to highlight a series of } \\
\text { reflections on the role of interdisciplinary } \\
\text { and transdisciplinary in generating } \\
\text { knowledge processes within universities. To } \\
\text { achieve a clear presentation, a brief story } \\
\text { related to a primitive way how humans } \\
\text { interacted with the environment was } \\
\text { presented, generating ancestral knowledge } \\
\text { that was part of the genesis of further } \\
\text { knowledge. Then, arguments aimed to } \\
\text { highlight theoretical and discursive } \\
\text { elements were developed in the } \\
\text { interdisciplinary and transdisciplinary } \\
\text { perspectives to understand the nature and } \\
\text { dynamic of them, what allowed configuring } \\
\text { a set of inputs whose aim is to serve for } \\
\text { debate and discussion of specific ideas } \\
\text { whose purpose is to open the possibility of } \\
\text { reaching total and not fragment knowledge. }\end{array}$ \\
\hline $\begin{array}{l}\text { Descriptores: Interdisciplinariedad, } \\
\text { transdisciplinariedad, universidad. }\end{array}$ & $\begin{array}{l}\text { Keywords: Interdisciplinary, } \\
\text { transdisciplinary, university. }\end{array}$ \\
\hline
\end{tabular}

\footnotetext{
* Profesor Ordinario, Dedicación Exclusiva. Estudios de IV y V Nivel. Universidad de Carabobo. Facultad de Ciencias de la Salud Escuela de Salud Pública y Desarrollo Social. Departamento de Ciencias Sociales.

Correo electrónico: rosmel.orfila.vilera@gmail.com
} 


\section{La universidad en la perspectiva de la interdisciplinariedad y transdisciplinariedad Rosmel Orfila ( Pp. 128-145)}

\section{INTRODUCCIÓN}

"El perfil monocromo del egresado le ciega a ver más allá de su disciplina. Con estas gríngolas los que sugieren o toman decisiones de impacto social pueden causar grave daño. Por esto la necesidad de una visión transdisciplinaria" Altschuler

El mundo científico tal como lo conocemos en este tiempo, ha transitado históricamente por diversos escenarios imprimiéndole rasgos que lo diferencia de otras maneras de explicar las cosas, es decir, como el misticismo. Hablar del conocimiento y de las diversas maneras a través de las cuales los seres humanos lo obtenemos, nos invita a la reflexión, además de seguir la búsqueda de explicaciones tendentes a poner de manifiesto la necesidad de que sea lo más acabado posible, como para dar razón del saber total que fundamenta la naturaleza y esencia del hombre dentro de su propio entorno sociocultural complejo.

La cuestión luce interesante cuando hay que considerar que el conocimiento es uno de los productos intelectuales de los seres humanos, teniendo presente que es, en esencia, connatural a la especie humana. Desde la época del hombre primitivo, las maneras de generar ciertas explicaciones estuvieron estrechamente ligados a los diversos acontecimientos de estirpe natural, en particular aquellos propios de las inclemencias del tiempo, de la búsqueda de alimentos, las maneras para protegerse de los animales silvestres, entro otros de interés para aquellos.

En esos primeros estadios de tiempo, que sirvieron para que los seres humanos se multiplicaran y migraran hacia cada rincón del planeta Tierra, el contacto directo con el entorno geográfico permitió comprender ciertas dinámicas de la naturaleza, como los ciclos de las lluvias, la relación estrecha entre estas y la agricultura, la importancia de los asentamientos cerca de las fuentes de aguas, hasta que progresivamente descubre la manera de dominar el fuego e inventa los artefactos líticos útiles para la caza, la pesca y la domesticación de animales.

Este breve relato intenta dibujar algunos rasgos que dominaron la escena humana en sus primeros momentos, permitiendo puntualizar que ya la generación de saberes es un hecho. El hombre primitivo fue consciente del comportamiento de sí mismo y del entorno, lo que 
dio paso a contemplarse como parte indisoluble de la naturaleza: hombre y ambiente es un "todo" infragmentable.

\section{HOMBRE, ENTORNO Y CONOCIMIENTO: UN TODO INDISOLUBLE}

El conocimiento debe concebirse como totalidad, y para lograr que sea percibido como un todo los escenarios académicos universitarios intentan configurar sistemas paradigmáticos tendentes al logro de la unidad del saber y conocimiento. Todo intento por procurar que el hombre de ciencia se aleje por completo del monismo metodológico, invita a cuestionar los conocimientos enmarcados en compartimentos estancos heredados, pues estos coartan cualquier intento por considerar las múltiples dimensiones y conexiones complejas en las que se ha movido históricamente el hombre, y en este sentido Martínez (2004), plantea que,

En la investigación monodisciplinaria enfatizamos la comprehensión o profundidad a expensas de la extensión. Nos quedamos dentro del ámbito de una sola disciplina. Puede llevarse a cabo por uno o varios investigadores que comparten plenamente un determinado paradigma científico: epistemología, métodos, técnicas y procedimientos. Es la más usual y corriente. Este enfoque lleva a aislar demasiado los elementos o las partes y su comportamiento, descuidando los nexos y relaciones que tienen con el todo y con otros "todos". En la hiperespecialización es donde más se puede revelar su exceso. (p. 1)

Reduccionismo de la realidad parece ser la norma de las disciplinas, lo cual trae consigo lo que el precitado autor define como la hiperespecialización. Este punto de vista es comprensible cuando se intenta lograr la comprensión de la esencia de los fenómenos, que juntos conforman las tramas complejas de la realidad y del hombre.

La disciplinariedad ha sido una consecuencia de la incesante búsqueda de respuesta a situaciones y comportamientos de la naturaleza, de inquirir sobre la biología, anatomía y fisiología del cuerpo humano, de saciar la curiosidad sobre el cosmos y, muy tímidamente, por estudiar las dimensiones socioculturales de las sociedades del viejo y nuevo mundo. Realizar las primeras disecciones al cuerpo humano durante la Edad Media, concretamente en Francia, representó un acto de herejía mal visto y castigado por la Iglesia Católica, 
recordemos que en esos estadios históricos el oscurantismo fue la norma, y las decisiones en todos los procesos y escenarios de la vida giraban en torno a los dictámenes del Papa.

Todos los cuestionamientos y sanciones pasaron por las manos de esa instancia religiosa, lo cual mantuvo inmóvil a toda una sociedad de manera insoslayable. Cuando Copérnico, Leonardo Da Vinci, entre otros, dirigieron sus miradas científicas para hurgar fenómenos propios de la naturaleza y del universo, sentaron las bases que lentamente dieron génesis a las disciplinas científicas. Todos los aportes generados desde el siglo XV, para precisar un tiempo, hasta los albores del siglo XIX, han sido decisivos para dar fisionomía a las ciencias que orbitan alrededor de las dimensiones de lo visible y cuantificable por excelencia. Esta perspectiva de producción científica fue decisiva, así como lo reafirma Gimeno (2002),

Podemos hablar con reserva de clasificaciones científicas a partir de la Edad Moderna, y específicamente, a partir del Renacimiento, con el surgimiento de las ciencias naturales como ciencia sistemática en la segunda mitad del siglo $\mathrm{XV}$. Las ciencias naturales constituyen el primer sistema científico por excelencia y es su objeto de conocimiento... (p. 13)

Sin embargo, ante la pléyade de compartimentos estancos que se gestaron históricamente hasta lograr alcanzar su florecimiento a mediados del siglo XV, también figuró como decisivo el pensamiento y saber filosófico, recordando los magistrales aportes de los filósofos presocráticos, hasta las contribuciones de Sócrates, Platón y Aristóteles desde el siglo IV A.C., entre algunos de los más notables.

Vale recordar aquí, que la filosofía abraza a la matemática, con su lógica formal y algunos elementos de física. Al respecto, Gimeno (2002) expone que,

El Estagirita (se refiere a Aristóteles) estableció tres categorías del saber: la «Theoría» o saber teórico, que atendía a la verdad de las ideas, su forma y sustancia: lo constituían las ciencias formales y experimentales cuyo conocimiento está basado en el saber por el saber: Matemáticas, Física y Teología; la «Praxis» o saber práctico encaminado al logro de un saber guía de la conducta humana: lo formaban la Ética, la Política, la Económica y la Retórica; y la «Poiesis» o saber creador, saber poético, basado en la transformación técnica o «Techné». (p. 13)

Fíjense que hay una riqueza de contribuciones de todo tipo, y estas se encuentran imbricadas en un contexto sociohistórico, fraguado en la reflexión y el repensar la manera 
de hacer las cosas en materia de investigación y de hacer ciencia. Cada estructura social busca transferir sus saberes, desplegándose así la episteme que se va permeando en el entretejido armazón de la ciencia, del hombre y de la sociedad en su conjunto.

\section{Cada campo disciplinar fragmenta el conocimiento}

El campo disciplinar que condicionó la hiperespecialización trajo consigo la fragmentación del conocimiento, que si bien tiene sus bondades, en algunos casos, es poco provechoso para otros. Según la óptica con la que miremos el asunto, hay notables intentos por hacer posible la integración disciplinar a la luz de generar conocimientos totales, integrales, es decir, que den razón de lo "dado" en completa interconexión con el todo. Dichos intentos unificadores dieron sus frutos con la aparición del paradigma interdisciplinar, que al decir de Martín-Barbero (2005),

La interdisciplinaridad implica una primera ruptura al trasladar métodos de una disciplina a otra, lo que afecta al estatuto de lo disciplinario en forma mucho más honda y fuerte, ya que ello viene a trastornar el funcionamiento de la disciplina. Pues lo que se introduce en ella es del orden epistémicometodológico y ya no del orden de la información. (p. 2)

¿El acento esta en el método? ¿Trasladarlo de una disciplina a otra con pretensiones de búsqueda de solución a problemas que no se les puede dar solución? ¿Es un intento desesperado por frenar la desaparición inevitable de disciplinas que no ofrecen nada nuevo? ¿Es el preámbulo a la configuración de la unicidad para hacer ciencia con productos totales? Estas son solo algunas inquietudes muy personales que buscan comprender como la disciplina por sí sola esta luchando por la supremacía de la producción científica mundial.

Para Neira (s/f), la interdisciplinariedad debe ser comprendida como,

... la interacción entre dos o más disciplinas diferentes. Esta interacción puede ir desde una simple comunicación de ideas hasta la mutua integración de conceptos, metodología, procedimientos, epistemología, terminología, datos y la organización de investigación y docencia en un campo suficientemente amplio. Un grupo interdisciplinario está compuesto de personas entrenadas en diferentes campos de conocimiento (disciplinas), con diferentes conceptos, métodos y datos, pero organizados en un esfuerzo común alrededor de un problema común, con intercomunicación continua entre los participantes que provienen de diferentes disciplinas. (p.6) 
La interdisciplinariedad es consustancial con el mundo complejo en su franco intento por insertarse dentro de las dimensiones ininteligibles del monismo metodológico. Esto nos invita a repensar la necesidad de abarcar con la mayor cantidad de aristas cada dimensión objeto de estudio en la tarea científica de la comprensión total de la complejidad. En atención a este aspecto, resulta interesante poner de manifiesto algunos planteamientos de Follari (2005), quien expone que,

Ya lo avanzado en el conocimiento de lo social no podría ser reconducido a un solo espacio de explicación. Sin embargo, la noción de totalidad como categoría organizadora de la mirada de cada disciplina, alcanza todavía sentido. Que cada una actúe sabiendo que su especificidad no existe y que solo responde a un recorte instrumental y analítico, permitiría dejar de pretender que cuando se hace economía a secas, se está haciendo ciencia suficientemente justificada, menos aún "exacta". (p. 2).

Todo apunta, entonces, a posibilitar espacios para la investigación científica total, integrada al mundo complejo que brinda oportunidades para ser escudriñado a través del prisma disciplinar de las ciencias. La tarea no es fácil, pues aun hay tensiones entre la mayor parte del conjunto de las parcelas disciplinarias.

Utopía o realidad, los intentos por alcanzar abordajes interdisciplinarios se hacen sentir en el advenimiento de una novísima mentalidad del sujeto que investiga más allá de su propia parcela científica. Sin embargo, no todo luce prometedor, pues, para Peñuela (2005), "Lo importante es advertir que la interdisciplinariedad ha avanzado solo como concepto, cuando en realidad es la articulación con la dimensión práctica la que puede determinar los alcances y limitaciones de esta.” (p. 22)

En este panorama, en el cual se conjugan las perspectivas filosóficas, las disciplinas en compartimentos aislados con la hiperespecialización como su nota dominante y la interdisciplinariedad como un intento por alcanzar el conocimiento total, se imbrica lentamente la Transdisciplinariedad de la que abordaré ahora.

La interdisciplinariedad asume diversas definiciones y perspectivas, y las mismas permiten afianzar que la misma constituye un esfuerzo por lograr que las disciplinas establezcan lazos de reciprocidad con el fin de aportar solucionar problemas susceptibles de ser abordados con ópticas científicas distintas. Para Grisolía, M. (2008), 
...en la interdisciplinariedad se involucran métodos y saberes de distintas disciplinas y se aplican a un problema determinad, el cual no concierne únicamente a una misma disciplina sino que pueden ser abordados mediante diversos ángulos o puntos de vistas... Es importante resaltar que mediante la vía interdisciplinaria no se ignoran las identidades propias de cada disciplina, sino que se entiende que cada una de estas puede realizar aportes importantes y diferentes entre si al conocimiento que puede constituirse acerca del sistema en estudio o del problema a solucionar. (p. 4)

Bien que se establezcan lazos comunicantes entre disciplinas, lo cual imprime a los procesos de investigación científica abordajes acabados, procuran con ello estudiar a profundidad dimensiones que solo pueden ser analizadas a través de la diversidad metódica y de conocimientos complementarios. La interdisciplinariedad debe ser concebida en los escenarios educativos, concretamente cuando se trata de enseñanza de las ciencias, de las artes; en las universidades, en los recintos de aula y en los centros de investigación, y en conjunto en la labor profesional en los distintos escenarios en los cuales hay que establecer nexos de colaboración entre disciplinas para dar respuestas certeras al sin fin de problemas por los cuales la sociedad atraviesa.

Considero que estos lazos de acercamiento entre las disciplinas solo es posible cuando los científicos tienen este conocimiento; actuar de manera aislada, como aun se puede apreciar en muchas universidades, es ignorar la necesidad de abrirse paso a campos de investigación mas amplios, en los cuales profundizar la multidimensionalidad de cada problema es nuestro norte.

\section{La transdisciplinariedad: mirada para hacer ciencia}

Nuevos tiempos marcan los inicios de cambios en materia de producción científica y, con ella, la manera en que el pensamiento del hombre de ciencia ha de enfrentarse con las exigencias de un entorno cada vez más difícil de comprender, que reclama de mentes que trascienda las fronteras del conocimiento dado. La posibilidad de lograrlo ha llegado, y la Transdisciplinariedad se hace presente tanto en las discusiones en los ámbitos universitarios así como en algunos intentos por hacerse realidad. 
Nuestras universidades se han convertido en ricos escenarios en los cuales los temas alusivos a la producción científica se convierten en la nota dominante, y estas brindan la oportunidad para idear y concretar estrategias metodológicas que estén destinadas a penetrar la esencia de los fenómenos complejos. Estos últimos deben ser abordados por una mentalidad igualmente compleja, la cual permita asimilarse a la luz de una dialógica en franca concreción.

En concordancia con este último aspecto, Morin, Ciurana y Motta (2006), hacen planteamientos interesantes cuyas pretensiones permiten despejar dudas acerca de la naturaleza compleja de todo lo que nos rodea. Así, estos autores ofrecen algunas tentativas de respuestas ante la pregunta ¿Qué es la complejidad?, a lo que responden con los siguientes aportes,

A primera vista es un tejido de constituyentes heterogéneos inseparablente asociados, que presentan la paradójica relación del uno y lo múltiple. La complejidad es efectivamente el tejido de eventos, acciones, interacciones, retroacciones. Determinaciones, azares, que constituyen nuestro mundo fenoménico. Así es que la complejidad se presenta con los rasgos perturbadores de la perplejidad, es decir de lo enredado, lo inextricable, el desorden, la ambigüedad y la incertidumbre. (p. 54).

Ese es el mundo, fenomenoménico, que interesa en la investigación científica, que no se fragmente en parcelas o unidades de análisis para ser repartidos a la constelación disciplinar científica para su estudio, generando cada una parte de un conocimiento no total. Para contrarrestar la fragmentación del conocimiento surge otra alternativa paradigmática cuyas pretensiones es lograr la unidad total del mismo, y al respecto Martínez (2005) expone el alcance de otra óptica epistémica, advirtiendo que,

La investigación transdisciplinaria (mucho más reciente, escasa y difícil que las anteriores) va más allá de ellas, y les añade el hecho de que está constituida por una completa integración teorética y práctica. En ella, los participantes transcienden las propias disciplinas (o las ven solo como complementarias) logrando crear un nuevo mapa cognitivo común sobre el problema en cuestión, es decir, llegan a compartir un marco epistémico amplio y una cierta meta-metodología que les sirven para integrar conceptualmente las diferentes orientaciones de sus análisis... (p. 1)

De lo que se trata es de comprender que estamos en presencia de tiempos nuevos que condicionan cambios en la manera de actuar ante la complejidad; romper fronteras en los 


\section{La universidad en la perspectiva de la interdisciplinariedad y transdisciplinariedad Rosmel Orfila ( Pp. 128-145)}

saberes históricamente consolidados hacia observar detenidamente los nexos dimensionales, la diversidad, lo insignificante, lo subjetivo, lo cotidiano, lo no visible.

¿Qué aportes deben generar nuestras universidades para dar respuestas a las necesidades de la compleja realidad de las sociedades?, y ¿qué reclaman estas a las instituciones de educación universitaria? He aquí algunas interrogantes que deben ser respondidas, y es precisamente en estas donde hay que centrar cualquier intento de explicación que este dirigido a exponer el estatus y rol preferencial de las instituciones universitarias

Una tentativa de aproximación que ayuda a perfilar puntos de vista hacia la concreción de ideas factibles para que la universidad se vincule armónicamente con la sociedad esta en la praxis de la Transdisciplinariedad, la que se ha venido convirtiendo en una tentativa que traerá consigo el advenimiento de toda una cultura de trabajo, de enseñanzas, de aprendizajes que redundarán inevitablemente en una nueva academia.

Romper con la atomización de las ciencias "duras", cuyas directrices se desprenden del monismo metodológico como base del Positivismo a través de su único método, el Hipotético Deductivo, debe ser la perspectiva del nuevo hombre de ciencia que tenga pretensiones de traspasar las barreras disciplinarias con miras a lograr una integración entre aquellas disciplinas y las fundamentadas en enfoques enteramente cualitativos. La Universidad, en este sentido, tiene una enorme participación en esta materia y asumir dicha tarea se convierte en el norte de su acción.

Hurgando minuciosamente algunas consideraciones que al respecto la Carta de la Transdisciplinariedad del Convento de Arrábida (Noviembre, 1994) ha expuesto, he podido conseguir elementos que permiten tener a disposición argumentos críticos que reflejan claramente la realidad educativa que desde finales del siglo XX se ha fomentado.

Así, es ilustrativo el preámbulo de dicha carta, y en ella encontramos los siguientes fundamentos:

(a) La proliferación actual de las disciplinas académicas y no-académicas conducen a un crecimiento exponencial del saber que hace imposible toda mirada global del ser humano. (b) Solo una inteligencia que de cuenta de la dimensión planetaria de los conflictos actuales podrá hacer frente a la complejidad de nuestro mundo y al desafío contemporáneo de la autodestrucción material y espiritual de nuestra especie. (c) La vida esta seriamente amenazada por una tecnociencia triunfante, que solo obedece a la lógica horrorosa de la 
eficacia por la eficacia. (d) La ruptura contemporánea entre un saber cada vez mas acumulativo y un ser interior cada vez mas empobrecido conduce a un ascenso de un nuevo oscurantismo, cuyas consecuencias en el plano individual y social son incalculables. (e) El crecimiento de los saberes, sin precedentes en la historia, aumenta la desigualdad entre aquellos que lo poseen y los que carecen de ellos, engendrando así desigualdades crecientes en el seno de los pueblos y entre las naciones de nuestro plantea. (f) Al mismo tiempo que todos los desafíos enunciados tienen su contraparte de esperanza y que el crecimiento extraordinario de los saberes puede conducir, a largo plazo, a una mutación comparable al pasaje de los homínidos a la especie humana.

Fijémonos, detenidamente, en cada uno de los aspectos e ideas concretas expuestas en la precitada carta, y apreciaremos que reflejan la preocupación por la inminente amenaza de la vida en el planeta Tierra, máxime cuando la producción del hombre de ciencia ha traspasado los límites del conocimiento. La diversidad disciplinar, y la cada vez mayor especialización, hace que las ciencias duras como la química, la física, la biología, la astronomía, entre otras, adquieran un lugar preferencial en el concierto de las disciplinas de este tipo.

El resultado de la diatriba entre las disciplinas científicas ha sido el aislamiento del conocimiento, siendo para los seres humanos una constante que desemboca en percibir el conocimiento como un producto complejo y obtenido de manera fragmentado. Sin embargo, tal como lo expone Esser (2005), esa forma de entender y hacer ciencia es característica de la modernidad ya en decadencia, pues la nueva época ¿postmoderna?, ya está fraguando las bases que dan génesis a estilos de pensamientos contrapuestos a las viejas formas de percibir la realidad, siendo la transdisciplinariedad una de ellas, y la que promete cambios sustanciales y posibilidades para que el hombre comprenda su mundo.

La transdisciplinariedad constituye una asombrosa ambición para muchos hombres de ciencia preocupados; particularmente, considero un fin alcanzable y necesario para dar respuesta con aportes que fijen su mirada en contextos casi relegados hasta el momento y que obstaculizan que todas estas perspectivas sean exitosas: el aprovechamiento de los aportes de todos los que hacemos vida académica y de investigación, sobre todo de quienes nos desarrollamos en las ciencias sociales, echadas a un lado casi por completo por los científicos de las ciencias duras. 
Estas apreciaciones puntuales, invita a que tengamos claro el alcance de la transdisciplinariedad, concretamente en lo que respecta a su naturaleza. Una exposición interesante es la presentada por Martínez (2005), quien explica que,

... los estudios transdisciplinarios o metadisciplinarios; es decir, estudios que ponen el énfasis, respectivamente, en la confluencia de saberes, en su interacción e integración recíprocas, o en su transformación y superación...es el deseo de que el pensamiento transdisciplinario alimente en lo sucesivo la nueva visión de la Universidad. Su intención es hacer revolucionar la Universidad hacia un estudio de lo universal en el contexto de una aceleración sin precedentes de los saberes parcelarios; y consideran que cada evolución es inseparable de la búsqueda transdisciplinar, es decir, de lo que existe entre, a través y más allá de todas las disciplinas particulares. ... Este tipo de investigación es, sobre todo, un ideal muy escasamente alcanzado hasta el momento. (p. 3)

¿De qué sirven que los centros académicos y científicos generen y condensen los conocimientos para lograr sistematizarlos, hasta alcanzar configurarse productos acabados como lo representan las ciencias de hoy? Acumular ingentes productos científicos sin considerar e involucrar abordar al sujeto complejo imbricado dentro de una enmarañada red multidimensional, es negar la esencia y el deber ser de la ciencia, la tecnología y la academia en su conjunto.

La manera de hacer ciencia "sin conciencia", a título muy personal, tiene repercusiones éticas y bioéticas que constituyen parte de la deshumanización del hombre. Estas apreciaciones son válidas para todos los aspectos de la vida del hombre, y de los procesos propios de las ciencias desde los recintos académicos. En este sentido, Roldán (2008) plantea que,

...., afirmamos que hoy en día, no se concibe una ciencia que no se relacione con la Bioética y con otras áreas de conocimiento, propiciando un diálogo transdisciplinar que permita una visión integral. ..., facilita determinar las razones de los comportamientos en la práctica de la profesión, los principios que regulan dichas conductas, las motivaciones, los valores del ejercicio profesional, los cambios y las transformaciones a través del tiempo. (p.3)

La ética, en el sentido general de la norma a seguir, y la bioética en el ámbito de las ciencias y del hombre, buscan normar la injerencia de la ciencia en los diversos escenarios de la vida de los seres humanos. Esto es una respuesta inevitable cuando se habla de ciencia 
y de investigación científica, pues se esta ante una cuestión de importancia: el hombre que hace la ciencia y la tecnología es sujeto y objeto de conocimiento y de investigación.

Así, la definición de transdisciplinariedad queda entendida bajo dichas apreciaciones expuesta, tal como lo expone Nicolescu (1996) citado por Roldán (2008), al expresar que,

La transdisciplinariedad, por su parte concierne, como lo indica el prefijo "trans", "...a lo que simultáneamente es entre disciplinas a través de las diferentes disciplinas y más allá de toda disciplina, su finalidad es la comprensión del mundo presente, uno de cuyos imperativos es la unidad del conocimiento. (p. 3)

Comprender el presente, lo cotidiano del hombre, a través de la acción de la ciencia toda vez que el acento debe estar en develar los elementos socioculturales que dinamizan su accionar en un vasto escenario social, permite aclarar las causales y consecuencias de los diversos problemas que atraviesan las sociedades en los momentos actuales, y de qué manera pueden posibilitarse alternativas de ayuda de los mismos. Tomando como punto de referencia los planteamientos expuestos, resulta interesante preguntarse si ¿la "mirada" desde ópticas disciplinarias adversas se han conjuga para develar la esencia que subyace en los fenómeno y, a su vez, resolver los problemas por los que atraviesa la humanidad? Esta perspectiva interdisciplinaria ha tratado tímidamente por imponerse, y la misma ha servido para reflexionar sobre la relevancia de la ciencia para tratar globalmente los problemas sociales.

A estas alturas del debate, sobre los temas inherentes a la disciplina, interdisciplinariedad y transdisciplinariedad, es comprensible que en el campo de la investigación social hace falta que los aportes de las disciplinas científicas ayuden a elevar significativamente la calidad de vida que solo es alcanzable si se conjugan esfuerzos tendentes a una mejor educación dirigida hacia la vida. Para Oraisón (2000),

..., el desarrollo del currículo debe contemplar, además de los ritmos de aprendizajes propios de cada nivel, cuestiones sociales conflictivas, problemáticas actuales y cotidianas, que demandan a cada sujeto una toma de posición frente a determinadas situaciones complejas. Este es uno de los desafíos más importante de la educación en un mundo contemporáneo, donde los cambios se producen a un ritmo de aceleración geométrica, donde los avances científicos y tecnológicos en el último siglo han expandido los límites de la naturaleza humana y han modificado substancialmente la relación del hombre con su entorno, donde las sociedades se han vuelto cada vez más 


\section{La universidad en la perspectiva de la interdisciplinariedad y transdisciplinariedad}

Rosmel Orfila ( Pp. 128-145)

complejas y dinámicas y la cultura se debate entre la globalización y las identidades étnicas particulares. (p.3)

Una muestra más de la innegable necesidad de hacer posible que la acción educativa este al servicio del hombre, para que le proporcione las herramientas intelectuales indispensables que le permita enfrentarse de manera diligente y exitosa a la complejidad de una sociedad que demanda una acción antrópica inteligente, esto es, capaz de crear, inventar, proponer alternativas viables de interactuar con éxito.

\section{Un panorama esperanzador de la ciencia}

Sin embargo, todo luce esperanzador, y así debe ser; pero ante el panorama complejo que avizoramos a diario ¿Cómo hacer de la transdisciplinariedad una realidad en los diversos escenarios académicos y de la ciencia en su conjunto? ¿Qué podemos hacer para ir más allá de las disciplinas autónomas? ¿Cómo conjugar la generación de conocimientos necesarios por cada ciencia, que es la esencia y deber ser de los científicos y, a su vez, cómo hacer posible que cada disciplina científica brinde la posibilidad de servir de fundamento para hacer del hombre un sujeto que recobre su dignidad humana? ¿Cómo el hombre debe proceder para ser receptáculo de las bondades de la producción científica? ¿Qué características debe poseer la Universidad para propiciar la transdisciplinariedad? Estas interrogantes, y muchas otras que pueden sumarse, forman parte de mi preocupación como docente universitario, precisamente ante un panorama educativo que con urgencia reclama cambios profundos en esta materia.

Pues bien, comenzaré por exponer que en el ámbito científico de cada una de las disciplinas que juntas definen la ciencia como tal, los científicos se convierten en sujetos y objetos de conocimiento, y partiendo de esta premisa elemental, y de fácil comprensión, se convierten en el punto de partida de todo el asunto.

No me estoy refiriendo a que el hombre de ciencia renuncie a su praxis profesional y científica según su formación académica, solo que ocupe un espacio significativo para idear escenarios ricos en oportunidades donde sea posible la divulgación de visiones tendentes a abrir nuevos horizontes del quehacer de los humanos. Para ello, pensar de esta manera y, que se convierta en una realidad, es incuestionable advertir que a partir del recinto 
educativo de los estudios desde la educación inicial hasta la Universidad, haya un cambio de paradigma en los estilos de producción intelectual, de apropiación y difusión de saberes y de conocimientos. Cada centro escolar tiene la posibilidad de enriquecer los contenidos de los programas incorporando aquellos temas transversales que guarden estrecha relación con la vida, tales como aspectos inherentes "al ser, al hacer y al convivir".

Muchas han sido las críticas negativas que se le han atribuido a nuestra educación y, en este sentido, son válidas las apreciaciones de Oraisón (2000) quien expone claramente que,

Ante este panorama, la educación parece que no logra adaptarse adecuadamente a las transformaciones, donde los contenidos parecen discurrir por otro camino distanciado de la realidad, perdiendo impacto sobre los alumnos, en tanto no suscitan su interés, su motivación y ofrecen escasas posibilidades de transferencia. (p. 3)

Es esperanzador poder expresar que a través de nuestra labor docente tenemos inmensas posibilidades de propiciar cambios, orientados a hacer significativo el aprendizaje de los contenidos de las asignaturas que impartimos en las aulas y, al igual que nosotros, los científicos de las distintas áreas deben asumir con responsabilidad proceder acertadamente, esto es llevando a cabo uno de sus roles el cual esta precisamente en educar de manera integral.

Nuestro verbo, acción y compromiso se convierten en las herramientas estratégicas que bien pueden fomentar en los otros cambios hacia la concienciación colectiva; es una tarea que solo precisa de la intención por despertar en las masas el deseo de vivir con sentido humano, esto es con responsabilidad, armonía, deseos de superación, hacer las tareas asignadas con pericia, proyectarse hacia un futuro alcanzable partiendo de una actitud emprendedora.

Para alcanzar la tan anhelada concienciación colectiva, debemos iniciar por asumir la responsabilidad por difundir los aportes de las reflexiones generadas en los círculos académicos, creando espacios que involucren la participación de los estudiantes y docentes de distintas disciplinas, con un objetivo claro: "dialogar" para apreciar las bondades de las perspectivas disciplinarias para solucionar problemas que conciernen a todos.

El acento debemos ponerlo no solo en reconocer la importancia de los canales comunicantes entre las disciplinas, necesario para detener la fragmentación del 
conocimiento contrarrestando así el reduccionismo tan marcado, sino también para dirigir la mirada más allá de estas. Es decir, concienciar a los universitarios en la necesidad de abrazar el mundo complejo como una unidad, acercándonos con todo el arsenal de métodos con miras a construir una matriz epistémica que involucre a los seres humanos, su entorno natural y, lógicamente, los aportes científicos para dar razón de lo dado.

Como una reflexión muy personal, invito a los docentes y estudiantes de nuestras universidades reivindicarse como protagonistas del quehacer social, entendiendo que la Universidad debe conectarse a ese ámbito, y entender que somos sujetos y objetos de nuestras propias investigaciones; por tanto, no debemos actuar de manera irracional y aislada con nosotros.

\section{A MANERA DE CONCLUSIÓN}

Como se puedo apreciar, no hay fórmulas mágicas para activar mecanismos académicos tendentes a dar respuestas, y hacer de la transdisciplinariedad una realidad en los escenarios académicos y centros de investigaciones científica y tecnológica. Todo se irá desarrollando sobre la marcha, y el hecho de que hoy se aborde en las universidades estos aspectos es un comienzo seguro enrumbado hacia escenarios abiertos a la apertura.

Todos aquellos quienes de una u otra manera tenemos la responsabilidad de fomentar estilos de pensamientos críticos en poblaciones estudiantiles en los escenarios de educación formal universitaria, y en aquellos contextos no formales de la sociedad, tenemos la posibilidad de planear la labor docente hacia la formación del ser, el saber, el hacer y el convivir. Contribuimos con el ser cuando dirigimos la mirada profesional hacia despertar sensibilidades de los estudiantes y del colectivo.

Las apreciaciones personales sobre este asunto, de interés muy particular y apasionado, invitan atreverse a generar espacios discursivos y de acción para que el diálogo se enriquezca con los saberes y aportes intelectuales de los distintos profesionales formados en el ámbito académico de nuestras universidades y centros de investigación científica. Es contrarrestar las barreas que imposibilitan percibir y apreciar la naturaleza y dinámica del “mundo complejo" en el cual nos movemos. 
Tal complejidad requiere que nuestra concepción de pensamiento este abierta a la comprensión del mundo al que pertenecemos, aprovechando los aportes de todas las mujeres y hombres quienes estamos comprometidos con nuestra Universidad. Para clarificar un poco este panorama, Villegas y otros (2006), plantean algunas reflexiones interesantes al respecto:

... la sociedad se hace cada vez más compleja, por las diversas tendencias que conviven. Esta complejidad tiende a aumentar, al relacionarse diferentes niveles de realidad y, por lo tanto, se exige construir conocimientos correlativamente complejos. De allí que el enfoque de los problemas, el del reduccionismo, ha empezado a desmoronarse en la medida que surge y se difunda el nuevo enfoque de la complejidad, elaborado y aplicado con métodos transdisciplinarios. Se requiere una nueva racionalidad trenzada por las iniciativas, la cooperación, el sentido de responsabilidad, la capacidad de reaccionar unas cosas con otras, y así descubrir en todo momento los elementos emergentes de lo nuevo. Es decir, es necesaria una transformación del pensamiento en la elaboración de las construcciones mentales y su representación. (p. 21)

No podemos caer en el error de ignorar la riqueza de saberes y aportes de nuestros pares académicos, es la única vía, a mi parecer, de alcanzar los fines de la transdisciplinariedad como tentativa para generar conocimientos cada vez más acabados y necesarios para enfrentar los problemas complejos.

\section{REFERENCIAS}

Carta de la Transdisciplinariedad. Convento de Arrábida 6 de noviembre de 2004, 1994. Disponible: http://www.filosofia.org/cod/c1994tra.htm [Consulta2012, Agosto, 18]

Esser D., Joyce. (2005). La Transdisciplinariedad compleja como referente teórico para el abordaje del proceso - salud-enfermedad. En Investigación en Salud. Vol. VII. № 1. Abril. Valencia, Ediciones Medigraphip.

Follari, Roberto. (2005) La Interdisciplina revisada. En: Revista Andamios, Vol. 1, N². México.

Gimeno Parelló, Javier. (2002). De las clasificaciones ilustradas al paradigma de la Transdisciplinariedad. En EL CATOBLEPAS. Revista Crítica del presente. $\mathrm{N}^{\circ} 10$, diciembre 2002, página 13. Madrid - España.

Grisolía Cardona, Maricarmen. (2008). La transdisciplinariedad en la enseñanza de las ciencias. Artículo enviado para su publicación a la Revista Ciencia y Educación, 2008. 
Disponible en: http://www.learndev.org/dl/Innovación-udg-2002.pdf [Consulta: 2015, Enero]

Martínez Miguélez, Miguel. (2005). Transdisciplinariedad y Lógica Dialéctica. Un enfoque para la complejidad del mundo actual. Disponible http://prof.usb.ve/miguelm. [Consulta: 2012, Septiembre 9]

Martín-Barbero, Jesús. (2005). Transdisciplinariedad: notas para un mapa de sus encrucijadas cognitivas y sus conflictos culturales. En: Culturas, Identidades y Saberes fronterizos, J. E. Jaramillo (com.). CES, Bogotá.

Morin, Edgar, Ciurana, Emilio R. y Motta, Raúl D. (2006). Educar en la era planetaria. Barcelona, España: GEDISA

Neira Fernández, Enrique. (s/f). Interdisciplinariedad de la ciencia hoy. Disponible: http://www.webdelprofesor.ula.ve/cjuridicas/neira/pdf/ensayos/14interdisciplinariedad. pdf. [Consulta: 2012, Septiembre]

Oraisón, María M. (2000). La Transversalidad en la Educación Moral: Sus Implicancias y Alcance. Presentado en el Panel 2: "La transversalidad en el Currículo" del Foro Iberoamericano sobre "Educación en Valores" organizado por la Organización de Estados Iberoamericanos para la Educación, la Ciencia y la Cultura (OEI) en Montevideo, del 2 al 6 de octubre de 2000.

Peñuela Velásquez, Alejandro. (2005). Más allá de los conceptos, la dialéctica. En: Revista Andamios, Vol. 1, $\mathrm{N}^{\circ}$ 2. México.

Roldán P., María R. (2008). Bioética y Enfermería. Diálogo transdisciplinario. Disponible: Revista Electrónica de PortalesMédicos.com//www.portalesmedicos.com/publicaciones/artícles/1117/1/Bioética-y-EnfermeríaDiálogo-transdisciplinario.html. [Consulta: 2008, Junio, 16]

Villegas, Crisálida y otros. (2006) La Investigación: Un Enfoque Integrador Transcomplejo. Aragua: Universidad Bicentenaria de Aragua, Decanato de Investigación, Extensión y Postgrado 